\title{
Strategies for the Analysis of Poly(Methacrylic Acid) by Matrix-Assisted Laser Desorption/ Ionization Time-of-Flight Mass Spectrometry
}

\author{
Mark F. Wyatt, ${ }^{\mathrm{a}}$ Nicolas Schaeffer, ${ }^{\mathrm{b}}$ Bien Tan, ${ }^{\mathrm{b}}$ and Andrew I. Cooper ${ }^{\mathrm{b}}$ \\ ${ }^{a}$ EPSRC National Mass Spectrometry Service Centre (NMSSC), School of Medicine, Swansea University, \\ Swansea, United Kingdom \\ ${ }^{\mathrm{b}}$ Department of Chemistry, University of Liverpool, Liverpool, United Kingdom
}

We evaluated several aqueous-based sample preparation protocols for the analysis of poly(methacrylic acid) (PMAA) by matrix-assisted laser desorption/ionization time-offlight mass spectrometry (MALDI-TOFMS). The sample contained a pentaerythritol tetra(3mercaptopropionate) end-group, and was characterized in positive and negative ion modes using 2,5-dihydroxybenzoic acid (DHB) and 2,4,6-trihydroxyacetophenone (THAP) matrices. The major series observed were the $[\mathrm{M}+\mathrm{Na}]^{+}$species, in positive ion mode, and the $[\mathrm{M}-\mathrm{H}]^{-}$ species, in negative ion mode. The performance of DHB and THAP matrices was comparable in positive ion mode, but THAP outperformed DHB in negative ion mode. The use of ion-exchange beads (IXB) benefited the analysis, while the addition of sodium acetate ( $\mathrm{NaOAc}$ ) or trifluoroacetic acid (TFA) proved disadvantageous in positive ion mode. (J Am Soc Mass Spectrom 2007, 18, 1507-1510) (c) 2007 American Society for Mass Spectrometry

A lthough matrix-assisted laser desorption/ ionization time-of-flight mass spectrometry (MALDI-TOFMS) has been utilized predominately within the life sciences, there has been considerable use of this technique for the characterization of synthetic polymers. The large body of work in this area has been the subject of several reviews [1-3]. However, there are few examples of the characterization of poly(methacrylic acid) (PMAA) homopolymers by MALDI-TOFMS in the literature. The analytical protocols used in positive ion mode are 2,5-dihydroxybenzoic acid (DHB) matrix in methanol [4] or DHB and sodium trifluoroacetate in acetone [5]. Sinapinic acid in methanol $(\mathrm{MeOH})$ or water $\left(\mathrm{H}_{2} \mathrm{O}\right)$ has been used in negative ion mode [6]. Consistent throughout these data are very poor signal-to-noise ratios and non-isotopic resolution of the observed species.

The current investigation is concerned with the analysis of a low molecular weight PMAA sample containing a pentaerythritol tetra(3-mercaptopropionate) end group (PTMP-PMAA), shown in Figure 1, by MALDITOFMS. This polymer and similar ligands have been used as a ligand in the size-controlled synthesis of gold nanoparticles, which are only soluble in water $[7,8]$. The goal of the study was to establish aqueous-based sample preparation protocols that would provide data with improved signal-to-noise ratios and resolution

Address reprint requests to Dr. M. F. Wyatt, EPSRC National Mass Spectrometry Service Centre (NMSSC), School of Medicine, Swansea University, Swansea SA2 8PP, UK. E-mail: m.f.wyatt@swansea.ac.uk compared with existing methods. These protocols could subsequently be used for the characterization of the PMAA-coated gold nanoparticles [8].

\section{Experimental}

DHB and 2,4,6-trihydroxyacetophenone (THAP) matrices, and trifluoroacetic acid (TFA), were purchased from Fluka (Dorset, UK). Dowex 50W-X8, $200 \mu \mathrm{m}$, ion-exchange beads (IXB), di-ammonium hydrogen citrate (DAC), sodium acetate (NaOAc), and ammonium acetate were purchased from Sigma-Aldrich (Dorset, UK). IXB were loaded with $\mathrm{NH}_{4}^{+}$ions as reported previously [9]. HPLC grade acetonitrile (MeCN) and $\mathrm{MeOH}$, both purchased from Fisher Scientific (Loughborough, UK), and Milli-Q $\mathrm{H}_{2} \mathrm{O}$ (Watford, UK) were used where appropriate. PTMP-PMAA $\left(M_{\mathrm{n}}=1900 \mathrm{~g}\right.$ $\mathrm{mol}^{-1}$ and $M_{\mathrm{w}}=2240 \mathrm{~g} \mathrm{~mol}^{-1}$ by GPC) was synthesized as reported elsewhere [8].

DHB and THAP matrix solutions were made to a concentration of $10 \mathrm{mg} \mathrm{mL}^{-1}$ in $1: 1$ ( $\mathrm{vol} / \mathrm{vol}$ ) $\mathrm{H}_{2} \mathrm{O}$ / $\mathrm{MeCN}$. DHB solution was also made to the same concentration in $\mathrm{MeOH}$. Aqueous matrix solutions were mixed with DAC solution $\left(50 \mathrm{mg} \mathrm{mL}^{-1}\right.$ in $\left.\mathrm{H}_{2} \mathrm{O}\right)$ in a 9:1 ratio. PTMP-PMAA solution was made to a concentration of $10 \mathrm{mg} \mathrm{mL}^{-1}$ in $\mathrm{MeOH}$, and mixed with the matrix solutions in a $5 \mu \mathrm{L}: 50 \mu \mathrm{L}$ ratio. One $\mu \mathrm{L}$ of $\mathrm{NaOAc}$ solution $\left(10 \mathrm{mg} \mathrm{mL}^{-1}\right.$ in $\left.\mathrm{MeOH}\right)$ or $100 \%$ TFA was added as required. For IXB preparations, $\sim 0.5 \mathrm{mg}$ of $\mathrm{NH}_{4}^{+}$-loaded IXB was added to $200 \mu \mathrm{L}$ of the PTMPPMAA solution, which was agitated by vortex mixer (at 
<smiles>CCC1(C)C(C(=O)O)C2CCC1(C)C2(C)SCCC(=O)OCC(COC(=O)CCS)(COC(=O)CCS)COC(=O)CCS</smiles>

Figure 1. Structure of PTMP-PMAA polymer.

slow speed) for $20 \mathrm{~s}$, before mixing with matrix; $0.5 \mu \mathrm{L}$ of the final mixture was spotted onto a stainless steel sample plate and dried in a stream of cool air.

MALDI-TOFMS data were acquired using an Applied Biosystems Voyager DE-STR spectrometer (Framingham, MA), which is equipped with a nitrogen laser $(\lambda=337 \mathrm{~nm})$. The instrument was operated in positive or negative ion reflectron mode. The accelerating voltage was $20 \mathrm{kV}$, while the grid voltage was maintained at $65.5 \%$. The delay time was $150 \mathrm{~ns}$, and laser fluence was attenuated to just above the threshold of ionization, which varied between matrices and preparations. The laser was fired at a frequency of $3 \mathrm{~Hz}$, and spectra were accumulated in multiples of 25 laser shots, with 150 shots in total. Postacquisition processing of data were performed utilizing Data Explorer V4.0 software supplied by Applied Biosystems.

\section{Results and Discussion}

Several different sample preparation protocols were evaluated; $\mathrm{DHB}$ in $\mathrm{MeOH}\left(\mathrm{DHB}^{1}\right)$, $\mathrm{DHB}$ in $\mathrm{H}_{2} \mathrm{O} / \mathrm{MeCN}$ $\left(\mathrm{DHB}^{2}\right), \mathrm{DHB}^{2}+\mathrm{NaOAc}, \mathrm{IXB}+\mathrm{DHB}^{2}, \mathrm{IXB}+\mathrm{DHB}^{1}+$ TFA, THAP/DAC, and DHB/DAC. These strategies were based on existing methods that have proven useful for similar systems. Synthetic polymers are often ionized by cationization. The doping of sample preparations with metal salts to achieve more homogeneous cationization, rather than relying on ubiquitous impurities, has become common practice [10]. The THAP/ DAC preparation is used for low molecular weight oligonucleotides, with the $\mathrm{NH}_{4}^{+}$ions acting as a buffer preventing replacement of acidic protons by metal ions [11]. IXB have been used previously for the characterization of polymeric acids by negative ion mode MALDI-TOFMS [12]. TFA was also added to promote the acid form of the pendant groups, before ionization. The data acquired in positive ion mode will be considered first and is presented in Figure 2.

The observed distributions are very similar for all preparations, except when $\mathrm{NaOAc}$ was added, and for DHB/DAC where no polymeric series were observed. In general, two polymeric series were clearly observed and both are isotopically resolved. Average molecular weight values obtained for the spectra are consistently slightly lower that those obtained by GPC $\left(M_{\mathrm{n}} \approx 1500 \mathrm{~g}\right.$ $\mathrm{mol}^{-1}$ ), which may be due to the type of standard used to calibrate the GPC. The major series corresponds to the $[\mathrm{M}+\mathrm{Na}]^{+}$species, while the minor series is mass-shifted by $+16 \mathrm{Da}$, which could correspond to either the $[\mathrm{M}+\mathrm{K}]^{+}$species or the $[\mathrm{M}+\mathrm{Na}]^{+}$species of an oxidation product. Accurate mass measurement would be required to differentiate between these isobaric species. There were possibly two more series, but these were barely distinguishable from the background noise.

The observed isotope distributions of each species for both series extend 1 and 2 Da lower than the equivalent theoretical isotope distributions. The fractional masses of these "additional" ions are identical to the expected ions indicating two chemically similar species are overlapping, which differ by a single point of unsaturation. Disulphide bonds may form during the analysis of peptides that contain thiol groups, or the PTMP end group in this case. The observed polymeric

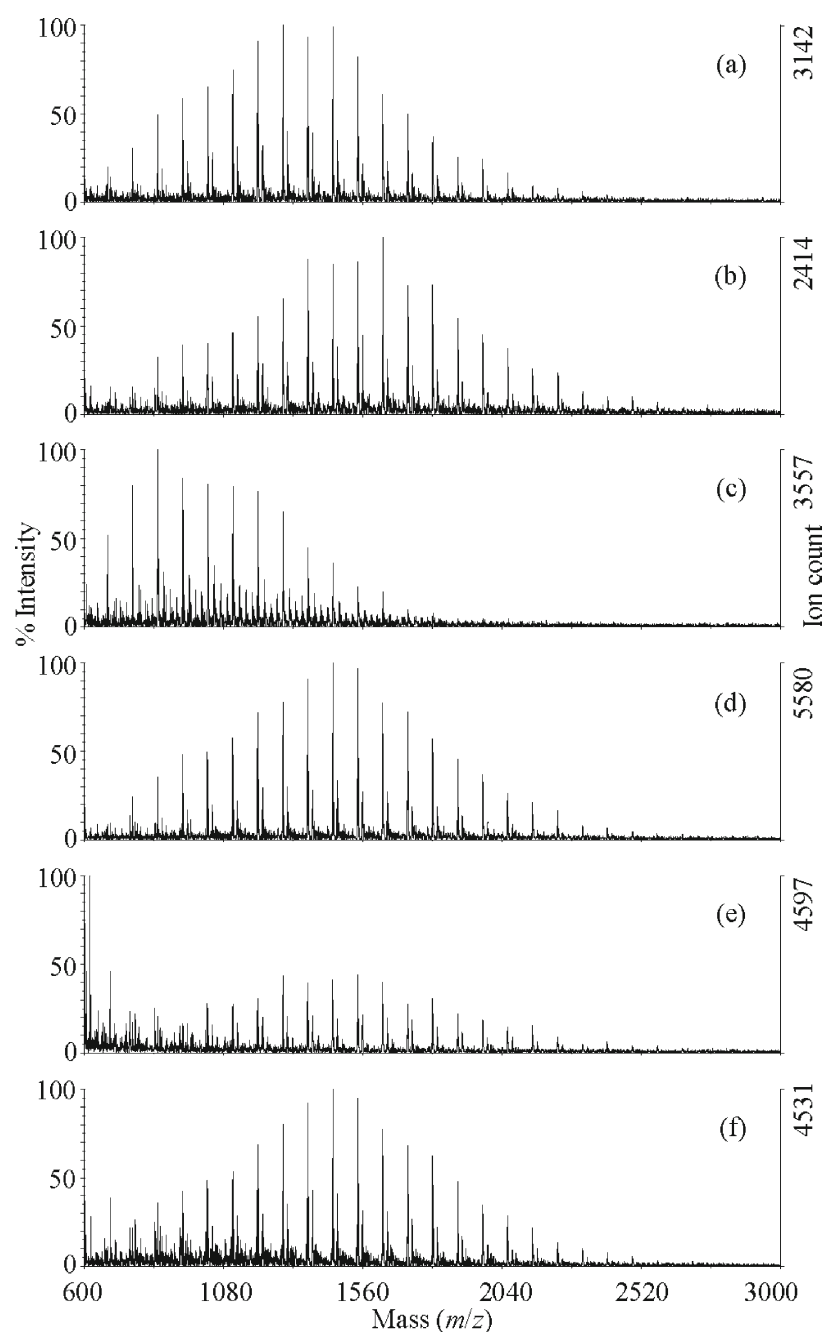

Figure 2. Positive ion MALDI-TOFMS data for PTMP-PMAA acquired with (a) $\mathrm{DHB}^{1}$, (b) $\mathrm{DHB}^{2}$, (c) $\mathrm{DHB}^{2}+\mathrm{NaOAc}$, (d) IXB + $\mathrm{DHB}^{2}$, (e) IXB $+\mathrm{DHB}^{1}+$ TFA, and (f) THAP/DAC sample preparation protocols. 
distributions are monomodal, and the relative intensities of the ${ }^{12} \mathrm{C}$-isotope for the expected and "additional" species remain approximately constant across the distribution, which both signify possible disulphide bonds would be intramolecular.

The use of IXB appears to have some benefit in improving the signal-to-noise ratio, but it is interesting that the major series observed is still $[\mathrm{M}+\mathrm{Na}]^{+}$. Good quality data were achieved with a significantly lower laser power compared with preparations without IXB. Little difference was observed between the spectra acquired with the $\mathrm{DHB}^{1}$ and $\mathrm{DHB}^{2}$ preparation methods. The addition of TFA was intended to promote protonation, which succeeded for low mass matrix or impurity species, but resulted in a reduction of the signal-to-noise ratio of the cationized polymer species. This result is contradictory to earlier studies of how $\mathrm{pH}$ affects the MALDI analysis of poly(methyl methacrylate) [13].

The addition of NaOAc resulted in a very different spectrum. The $[\mathrm{M}+\mathrm{Na}]^{+}$species continues to be the major series. There were three minor series also observed, which were successively mass-shifted by +22 Da. These series are likely to correlate to the $[\mathrm{M}+\mathrm{Na}]^{+}$ species for successive replacement of up to three acidic protons by $\mathrm{Na}^{+}$ions. There was a significant shift of the distribution towards the lower mass region $\left(M_{\mathrm{n}} \approx\right.$ $1200 \mathrm{~g} \mathrm{~mol}^{-1}$ ), and discrimination against higher mass species. Previous studies of cation attachment to polar polymers cannot account for this observation [13, 14]. A possible explanation is that the $\mathrm{NaOAc}$ interfered with the ability of DHB to desorb the larger polymer chains, leading to the equivalent of LDI spectrum, i.e., without the use of a matrix.

The data acquired in negative ion mode is presented in Figure 3. Again, no polymeric series were observed with DHB/DAC. No significant shifts of the polymer distribution or discrimination towards any mass region were observed between different sample preparations, and average molecular weight values were consistent with those obtained in positive ion mode. However, there were considerable differences in the relative intensities of the species observed. The major series in all the spectra were the $[\mathrm{M}-\mathrm{H}]^{-}$species. There were at least four minor series observed; two series are massshifted by +22 and +38 Da from the major series and are likely to correspond to the $[\mathrm{M}-2 \mathrm{H}+\mathrm{Na}]^{-}$and $[\mathrm{M}-2 \mathrm{H}+\mathrm{K}]^{-}$species. Another series could correspond to the loss of $\mathrm{H}_{2} \mathrm{O}$, but these species are not of sufficient intensity to confirm this assignment. Other series were observed clearly with the $\mathrm{DHB}^{2}$ and IXB + $\mathrm{DHB}^{1}+$ TFA protocols, mass-shifted by -30 or $+56 \mathrm{Da}$, and with the IXB $+\mathrm{DHB}^{2}$ protocol, mass-shifted by -34 or $+52 \mathrm{Da}$, but their origins are ambiguous.

The use of IXB appears to enhance deprotonation and/or suppress replacement of acidic protons by metal ions, increasing the signal-to-noise ratio of the major series. Again, good quality data were achieved with a significantly lower laser power compared with

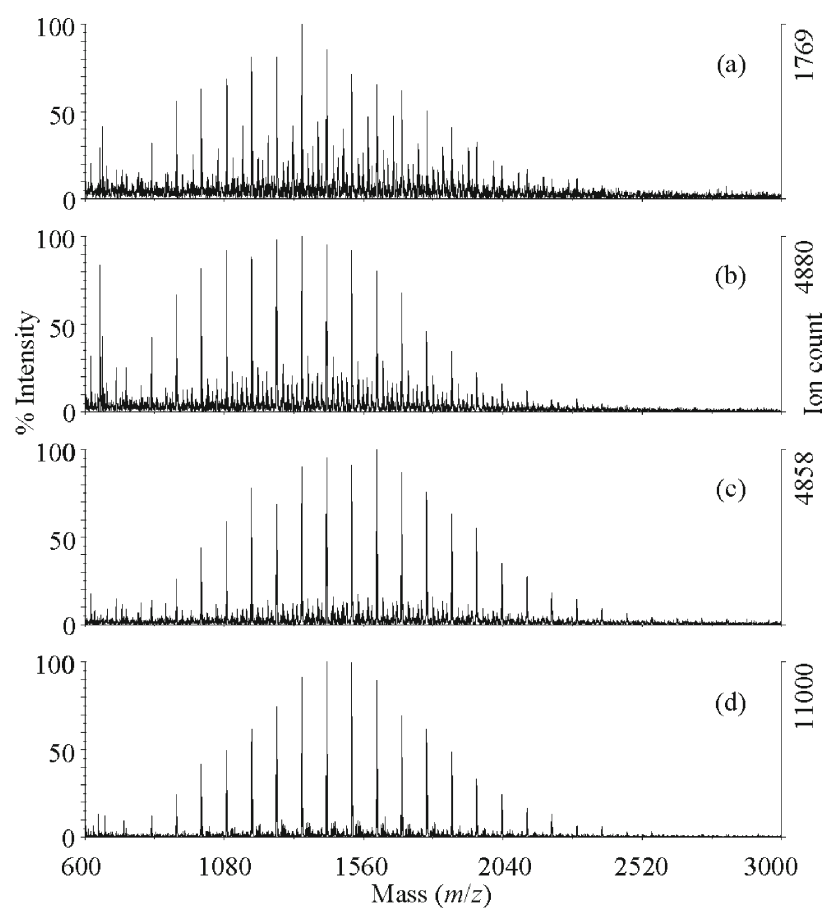

Figure 3. Negative ion MALDI-TOFMS data for PTMP-PMAA acquired with (a) $\mathrm{DHB}^{2}$, (b) IXB $+\mathrm{DHB}^{2}$, (c) IXB $+\mathrm{DHB}^{1}+\mathrm{TFA}$, and (d) THAP/DAC sample preparation protocols.

preparations without IXB. There is possibly a slight further enhancement and/or suppression when TFA is added, but the most significant increase in signal-tonoise ratio was observed when the THAP/DAC method was used. This benefit must be balanced against the loss of potentially useful information of other species within the sample. The ions in each series were isotopically resolved and the "additional" ions observed 1 and 2 Da lower than the equivalent theoretical isotope distributions in positive ion mode were also observed. There is increased confidence that these "additional" ions are not artifacts, even if they are a product of the MALDI-TOFMS process.

\section{Conclusions}

The study of several aqueous-based sample preparation methods for PMAA has resulted in significant improvements in the quality of MALDI-TOFMS data. Greater signal-to-noise ratios were observed in both positive and negative ion modes when treating the sample solution with IXB, before mixing with the matrix. The $[\mathrm{M}+\mathrm{Na}]^{+}$species was the major series observed in positive ion mode, even when IXB were used, and the performance of DHB and THAP matrices was comparable. The addition of NaOAc or TFA proved disadvantageous in positive ion mode. Varying the concentration of metal salts added to a preparation is a possible avenue for further study. The major series in negative ion mode corresponded to the $[\mathrm{M}-\mathrm{H}]^{-}$species, and the THAP/DAC preparation produced significantly 
greater ion intensities than DHB. Maximum information can be gained by obtaining data in both positive and negative ion modes. These methods should prove to be widely applicable to polyacidic analytes.

\section{Acknowledgments}

The authors thank EPSRC (contract/grant numbers GR/R70088/01 and EP/C511794/1) for funding this work, and the Royal Society for a Royal Society Research Fellowship (to AIC).

\section{References}

1. Nielen, M. W. F. MALDI Time-of-Flight Mass Spectrometry of Synthetic Polymers. Mass Spectrom. Rev. 1999, 18, 309-344.

2. Murgasova, R.; Hercules, D. M. MALDI of Synthetic Polymers-An Update. Int. J. Mass Spectrom. 2003, 226, 151-162.

3. Montaudo, G.; Samperi, F.; Montaudo, M. S. Characterization of Synthetic Polymers by MALDI-MS Progress in Polymer. Science 2006, 31, 277-357

4. Saito, R.; Kobayashi, H. Synthesis of Polymers by Template Polymerization. 2. Effects of Solvent and Polymerization Temperature. Macromolecules 2002, 35, 7207-7213.

5. Saito, R.; Yamaguchi, K. Synthesis of Cyclic Methacrylic Acid Oligomers by Atom Transfer Radical Polymerization. J. Polym. Sci. A Polym. Chem. 2005, 43, 6262-6271.
6. Burkoth, A. K.; Anseth, K. S. MALDI-TOF Characterization of Highly Cross-Linked, Degradable Polymer Networks. Macromolecules 1999, 32, 1438-1444.

7. Hussain, I.; Graham, S.; Wang, Z. X.; Tan, B.; Sherrington, D. C.; Rannard, S. P.; Cooper A. I.; Brust, M. Size-Controlled Synthesis of Near-Monodisperse Gold Nanoparticles in the 1-4 nm Range Using Polymeric Stabilizers. J. Am. Chem. Soc. 2005, 127, 16398-16399.

8. Wang, Z. X.; Tan, B. E.; Hussain, I.; Schaeffer, N.; Wyatt, M. F.; Brust, M.; Cooper, A. I. Design of Polymeric Stabilizers for Size-Controlled Synthesis of Monodisperse Gold Nanoparticles in Water. Langmuir 2007, 23, 885-895.

9. Nordhoff, E.; Ingendoh, A.; Cramer, R.; Overberg, A.; Stahl, B.; Karas, M.; Hillenkamp, F.; Crain, P. F. Matrix-Assisted Laser Desorption Ionization Mass-Spectrometry of Nucleic-Acids with Wavelengths in the Ultraviolet and Infrared. Rapid Commun. Mass Spectrom. 1992, 6, 771-776.

10. Bahr, U.; Deppe, A.; Karas, M.; Hillenkamp, F.; Giessmann, U. MassSpectrometry of Synthetic-Polymers by UV Matrix-Assisted Laser Desorption Ionization. Anal. Chem. 1992, 64, 2866-2869.

11. Pieles, U.; Zurcher, W.; Schar, M.; Moser, H. E. Matrix-Assisted LaserDesorption Ionization Time-of-Flight Mass-Spectrometry-A Powerful Tool for the Mass and Sequence-Analysis of Natural and Modified Oligonucleotides. Nucleic Acids Res. 1993, 21, 3191-3196.

12. Danis, P. O.; Karr, D. E. Analysis of Poly(Styrenesulfonic Acid) by Matrix-Assisted Laser-Desorption Ionization Time-of-Flight MassSpectrometry. Macromolecules 1995, 28, 8548-8551.

13. Dogruel, D. Nelson, R. W. Williams, P. The Effects of Matrix $\mathrm{pH}$ and Cation Availability on the Matrix-Assisted Laser Desorption Ionization Mass Spectrometry of Poly(Methyl Methacrylate). Rapid Commun. Mass Spectrom. 1996, 10, 801-804.

14. Jackson, A. T.; Yates, H. T.; MacDonald, W. A.; Scrivens, J. H.; Critchley, G.; Brown, J.; Deery, M. J.; Jennings, K. R.; Brookes, C. Time-Lag Focusing and Cation Attachment in the Analysis of Synthetic Polymers by Matrix-Assisted Laser Desorption Ionization Time-of-Flight Mass Spectrometry. J. Am. Soc. Mass Spectrom. 1997, 8, 132-139. 